Preventive Care in Nursing and Midwifery Journal

2020; 10(1): 39-45

\title{
The role of reproductive variables, anxiety, physical activity, on the sleep quality of lactating women referring to health care centers of Zanjan-Iran
}

\author{
Youseflu $\mathrm{S}^{1} \oplus$, Bayat $\mathrm{Z}^{2} \oplus$, Amiri $\mathrm{F}^{3} \oplus$, Mohebbi $\mathrm{P}^{1^{*}}$ (i) \\ ${ }^{* 1}$ Instructor, Department of Midwifery, School of Nursing and Midwifery, Zanjan University of Medical Sciences, Zanjan, Iran \\ ${ }^{2}$ MSc, Student of midwifery at Tehran University of Medical Sciences, Tehran, Iran \\ ${ }^{3} \mathrm{BSc}$, Midwifery in Ayatollah Mousavi Hospital in Zanjan, Zanjan, Iran
}

*Corresponding Author Adress: Zanjan university of medical sciences \& health, Nursing and Midwifery faculty, Mahdavi st, Zanjan, Iran

Tel: 0098-9124424883

Email: p.mohebbi11@gmail.com

Received: 25 Aug $2020 \quad$ Accepted: 6 Oct 2020

\begin{abstract}
Background: Mothers in the postpartum period experiences a change in sleep pattern and loss of sleep duration. In the meantime, factors can cause irregular sleep patterns and decreased sleep quality.

Objectives: The aim of this study was investigating the factors of fertility, anxiety and physical activity on the sleep quality of the lactating women visiting healthcare centers in Zanjan, Iran.

Methods: This descriptive cross-sectional study was carried out from 2018 to 2019 on 380 women aged 1445 years referred Ayatollah Mousavi Hospital in Zanjan and its affiliated clinics who were maximally 6 months postpartum and selected by convenience sampling method. The measuring tools used in this study included Petersburg Sleep Quality Questionnaire, Beck Anxiety Inventory, and a checklist containing demographic-fertility. Data were analyzed through SPSS software using descriptive and analytical tests (ttest, Mann-Whitney, and Chi-square). The statistical significance level was considered as $\mathrm{p}<0.05$.

Results: in this study $45.8 \%(n=174)$ of the participants suffered from mild anxiety and $13.2 \%(n=50)$ of them from moderate anxiety. The mean score of their sleep quality was $4.70 \pm 3.06$. The effect of physical activity $(\mathrm{p}$-value $=0.006)$ and anxiety level $(\mathrm{p}$-value $=0.002)$ on women's sleep quality was statistically significant.

Conclusion: The results of the present study confirm the positive effect of regular physical activity and the negative effects of anxiety on sleep quality of the breastfeeding mothers.
\end{abstract}

\section{Keywords: sleep quality, postpartum, anxiety, physical activity, reproductive variables}

\section{Introduction}

Sleep is a physiological need that relieves physical and mental fatigue and is an important need in the postpartum period for mothers to adapt to their new role [1]. Mothers in this critical period experience changes in their sleep patterns in that their sleep is usually interrupted at least once during the night and face an increase in nocturnal awakenings for the care and feeding of their infants [2]. Poor sleep quality and postpartum fatigue are two pressing and persistent problems facing new mothers [3]. Poor sleep quality may persist for as long as 12 months or even more after childbirth and its persistence and severity cause various problems for these women and their families [4,5].

Postpartum emotional and physical changes, as well as hormonal changes such as decreased progesterone and melatonin levels, lead to postpartum sleep changes [5]. Also, changes in the social status and psychological well-being of mothers that occur during this period are factors affecting the quality of sleep of mothers, so that the anxiety and mood disorders that occur in the postpartum period are among the risk factors for poor sleep during this period [6]. Anxiety 
disorders are prevalent during pregnancy and the postpartum period, and, in some cases, are more prevalent than depression. Children of mothers with anxiety disorders are at risk of adverse childhood consequences, such as affective, emotional and behavioral problems, as well as cognitive problems [7].

While poor sleep quality is an important indicator of maternal physical and mental health [8], and a better understanding of postpartum sleep and factors related to it can help facilitate the development of interventions to improve postpartum sleep quality [9], it has often been neglected by healthcare professionals Studies have shown that persistent sleep disturbances during the postpartum period can negatively affect maternal daily functioning, breastfeeding performance, maternal satisfaction, and motherinfant interaction. [1,9]. In a study conducted by Behboodi Moghaddam et al. on the quality of sleep in postpartum women, $62.5 \%$ of mothers had poor sleep quality and the frequency of awakening due to infant crying and the frequency of breastfeeding were the most important factors that were significantly associated with sleep disorders. [10].

Since limited research has been done in this regard [11] and in order to allocate health resources and reduce the negative impact of poor sleep quality on health outcomes, it is important to determine and examine the factors associated with this issue. [9] Therefore, the aim of the current study has been to investigate the effect of fertility factors, anxiety and physical activity on sleep quality of breastfeeding mothers visiting health centers in Zanjan, Iran in their postpartum period.

\section{Methods}

The present study is a descriptive cross-sectional study that examines the factors affecting the sleep quality of women in their postpartum period. This research has been carried out between 2018 and 2019 on 380 women who were maximally 6 months postpartum after obtaining the approval of the Ethics Committee in Medical Research (IR.ZUMS.REC.1398.112) and the approval of Zanjan University of Medical Sciences Research Vice Chancellor and carrying out administrative affairs related to obtaining the necessary permits from Ayatollah Mousavi Hospital officials and
Zanjan and comprehensive health service centers affiliated to Zanjan University of Medical Sciences. After examining the studies done in this regard [8], the prevalence of sleep disorders in the postpartum period was estimated at $28 \%$ and with certainty of $95 \%$ the number of samples required for each group is equal to the following formula:

$$
n=\frac{p \times(1-p) \times\left(z_{1-\frac{\alpha}{2}}\right)^{2}}{(d)^{2}}
$$

$\alpha$ : Probability of first type error, $\alpha=0.05$ and

$\mathrm{Z}_{1-\alpha / 2}=1.96$

$\mathrm{P}$ : Estimation of the proportion of the desired trait $\mathrm{P}=0.28$

$\mathrm{d}$ : Acceptable error in estimating the desired ratio $\mathrm{d}=0.04$

The number of samples required for this research is determined to be 380 people.

Written informed consent was obtained from all participants in the study, and they were assured that all the information they provide in the questionnaire would remain confidential.

Firstly, the selection was done using convenience sampling method among those women who referred Ayatollah Mousavi Hospital in Zanjan and its affiliated clinics between 2018 and 2019 and met the inclusion criterion of maximally 6 months postpartum.

Characteristics of the participants: Iranian race, experiencing no stressful incident in the last three months, literacy, consenting to study, being maximally 6 months postpartum. Initially, all the participants gave one-word answers to a list of questions related to their demographic-fertility status such as age, socio-economic status, employment status, education, cigarette smoking, number of pregnancies, number of deliveries, breastfeeding status, contraception method, and type of delivery. Participants were asked to rate their physical activity during the week. Their physical activity was classified into three levels: Level 1: Less than one hour a week, Level 2: between 1 to 3 hours a week, and Level 3: more than three hours per week.

Valid and reliable Pittsburgh Sleep Quality Questionnaire was completed by all participants. This is a self-report questionnaire that assesses sleep quality over a 1-month time interval, and consists of 19 items measuring several different aspects of sleep, offering seven component scores 
and one composite score. The component scores consist of 1 . Subjective sleep quality, 2. Sleep duration 3. Sleep latency, 4. Sleep disturbances, 5. Habitual sleep efficiency, 6. Use of sleeping medication, and 7. Daytime dysfunction.

Each item is weighted on a 0-3 interval scale. The global PSQI score is then calculated by totaling the seven component scores, providing an overall score ranging from 0 to 21 , where lower scores denote a healthier sleep quality [12]. Validity and reliability of Pittsburgh Sleep Quality Questionnaire (PSQI) has been reported in the study conducted by Pedram Razi et al as 0.86 and 0.89 respectively [13].

Valid and reliable Beck Anxiety Inventory questionnaire was completed to assess the level of anxiety of these women. The BAI contains 21 questions in the Likert scale, each answer being scored on a scale value of 0 (not at all) to 3 (severely). A score of 0-9 indicates normal anxiety level, 10-18 indicates mild anxiety level, 19-29 moderate anxiety and 63-30 severe anxiety [14]. The validity and reliability of this questionnaire has been confirmed in numerous domestic and foreign studies as well as the study conducted by Taqwi et al. Using Cronbach's alpha of 94.4 [15]. The data was analyzed using SPSS version 22.00. Kolmogorov- Smirnoff's (KS) test was used to check the normality of the data. Comparisons were made between two groups of women with good quality and poor sleep using ttest and Mann-Whitney test for quantitative data and Chi-square test for qualitative data.

\section{Results}

Out of a total of 380 women participating in this study, $72.37 \%$ (275) had a university education and $16.84 \%$ (64) were employed. The mean age of these women was $28.73 \pm 5.96$ years. Their main method of contraception was Withdrawal (48.16\%). $1.84 \%$ (7 people) of women were cigarette smokers but $17.89 \%$ (68 people) were passive smokers. $64.73 \%$ of women (246 women) had less than one hour of physical activity per week. $72.63 \%$ of the participants (276 women) had vaginal birth. $61.32 \%$ of the women (233 women) exclusively breastfed their baby, and $35.52 \%$ of them (135 women) mixed fed them (Table 1).

Table 1: Demographic Characteristics in Lactating Women

\begin{tabular}{|c|c|c|}
\hline \multicolumn{2}{|c|}{ characteristics } & $\mathrm{N}=\mathbf{3 8 0}$ \\
\hline \multicolumn{2}{|l|}{ Age } & $28.73 \pm 5.96$ \\
\hline \multicolumn{2}{|l|}{ Income } & $2.10 \pm 1.05$ \\
\hline \multirow{2}{*}{ Education } & More than 12 years & $275(72.37)$ \\
\hline & Less than 12 years & $105(27.63)$ \\
\hline \multicolumn{2}{|l|}{ Parity } & $1.72 \pm 0.90$ \\
\hline \multicolumn{2}{|l|}{ BMI } & $25.61 \pm 3.68$ \\
\hline \multirow{2}{*}{ Type of delivery } & NVD & $276(72.63)$ \\
\hline & $\mathrm{CS}$ & $104(27.37)$ \\
\hline \multirow{3}{*}{ Breast feeding } & Bottle feeding & $12(3.16)$ \\
\hline & Breast feeding & $233(61.32)$ \\
\hline & Both & $135(35.52)$ \\
\hline \multirow{4}{*}{ Contraception } & Hormonal & $112(29.47)$ \\
\hline & IUD & $64(11.58)$ \\
\hline & Condom & $21(5.52)$ \\
\hline & Withdrawal & $183(48.16)$ \\
\hline \multirow{3}{*}{ Physical activity } & Less than 1 hours per week & $246(64.73)$ \\
\hline & Between 2-3 per week & $98(25.80)$ \\
\hline & More than 3 hours per week & $36(9.47)$ \\
\hline \multirow{3}{*}{ Occupation } & Housewife & $207(54.47)$ \\
\hline & Self-employment & $109(28.68)$ \\
\hline & Employed & $64(16.84)$ \\
\hline \multirow{2}{*}{ Smoking } & Yes & $7(1.84)$ \\
\hline & No & $373(98.15)$ \\
\hline \multirow{2}{*}{ Passive smoker } & Yes & $68(17.89)$ \\
\hline & No & $312(82.10)$ \\
\hline
\end{tabular}


The sleep quality and anxiety levels of the women participating in the study has been reported in Table 2 . The results of the present study showed that $45.8 \%$ of the women (174 ones) had mild anxiety and $13.2 \%$ of them (50 ones) had moderate anxiety. The mean score of their sleep quality was $4.06 \pm 3.06$ (Table 2).

Table 2: Anxiety levels, PSQI total and component scores in lactating women

\begin{tabular}{ccc}
\hline & Subjective sleep quality & $0.99 \pm 0.86$ \\
\cline { 2 - 3 } Sleep quality & Sleep latency & $0.86 \pm 0.92$ \\
\cline { 2 - 3 } & Duration of sleep & $0.37 \pm 0.66$ \\
\cline { 2 - 3 } & Day time function & $0.18 \pm 0.43$ \\
\cline { 2 - 3 } & Sleep disturbance & $1.70 \pm 0.65$ \\
\cline { 2 - 3 } Anxiety & Sleep efficiency & $0.54 \pm 0.95$ \\
\cline { 2 - 3 } & Sleep medication & $0.05 \pm 0.28$ \\
\cline { 2 - 3 } & Total score & $4.70 \pm 3.06$ \\
\cline { 2 - 3 } & Normal & $147(38.7)$ \\
\cline { 2 - 3 } & Mild & $174(45.8)$ \\
\hline & Moderate & $5(13.2)$ \\
\hline
\end{tabular}

The difference in factors such as age, parity, type of delivery, exclusive breastfeeding, body mass index, and cigarette smoking between women with good and bad sleep quality was not statistically significant. Among these factors, physical activity $(\mathrm{p}$-value $=0.006)$ and anxiety level ( $p$-value $=0.002$ ) were the most important variables affecting the sleep quality of these women (Table 3).

Table 3: Quality of sleep among lactating women based on anxiety level, demographic, and reproductive characteristics

\begin{tabular}{|c|c|c|c|c|}
\hline \multicolumn{2}{|c|}{ Variable } & $\begin{array}{c}\text { Good sleeper } \\
(\mathrm{PSQI} \geq 5)(\mathrm{N}=\mathbf{2 2 3})\end{array}$ & $\begin{array}{c}\text { Poor sleeper } \\
(\text { PSQI }<5)(N=157)\end{array}$ & p-value \\
\hline \multicolumn{2}{|c|}{ Age } & $28.55 \pm 5.64$ & $28.98 \pm 6.39$ & 0.48 \\
\hline \multicolumn{2}{|c|}{ BMI } & $25.72 \pm 3.50$ & $25.43 \pm 3.93$ & 0.28 \\
\hline \multirow{2}{*}{$\begin{array}{l}\text { Type of } \\
\text { delivery }\end{array}$} & NVD & $164(73.54)$ & 112(71.33) & \multirow{2}{*}{0.63} \\
\hline & $\mathrm{CS}$ & $59(26.45)$ & $45(28.66)$ & \\
\hline \multirow{2}{*}{$\begin{array}{c}\text { Exclusive } \\
\text { breast-feeding }\end{array}$} & Yes & $143(65.92)$ & $90(57.32)$ & \multirow{2}{*}{0.18} \\
\hline & No & $80(35.87)$ & $67(42.68)$ & \\
\hline \multicolumn{2}{|c|}{ Parity } & $1.67(0.89)$ & $1.79(0.92)$ & 0.16 \\
\hline \multirow{2}{*}{ Smoking } & Yes & $2(2.24)$ & $5(3.18)$ & \multirow{2}{*}{0.10} \\
\hline & No & $221(97.76)$ & $152(96.81)$ & \\
\hline \multirow{3}{*}{$\begin{array}{l}\text { Physical } \\
\text { activity }\end{array}$} & Less than 1 hours & $129(57.85)$ & $117(74.52)$ & \multirow{3}{*}{0.006} \\
\hline & Between 2-3 hours & $71(31.84)$ & $27(17.20)$ & \\
\hline & More than 3 hours & $23(10.31)$ & $13(8.28)$ & \\
\hline \multirow{4}{*}{ Anxiety } & Normal & $103(46.19)$ & $44(28.02)$ & \multirow{4}{*}{0.002} \\
\hline & Mild & $89(39.91)$ & $85(54.14)$ & \\
\hline & Moderate & $28(2.56)$ & $22(14.01)$ & \\
\hline & Sever & $3(1.34)$ & $6(3.82)$ & \\
\hline
\end{tabular}

\section{Discussion}

The general aim of this study was to investigate the effect of factors affecting sleep quality of women in the postpartum period. According to the results of the current study, mother's average nighttime total sleep time in this period (mean score) was $3.064 .4 .70 \%$, in that $58.68 \%$ of mothers had good sleep quality and $41.31 \%$ had poor sleep quality. The highest index of low sleep quality observed during 6 months after delivery was related to sleep disturbance dimension $(0.651$ 0 1.70). In a study conducted in Taiwan, mothers' sleep quality was estimated to be $87.5 \%$ during the 12 months after delivery [16]. According to the study done by Wen et al, the prevalence of poor sleep quality in 3 months after the delivery among mothers aged 35 years and above was $(61.6 \%)$ and that of mothers aged less than 35 years was $(38.4 \%)$ [17]. In the study done by $\mathrm{Wu}$ et al., $59 \%$ of mothers reported poor sleep quality in the postpartum period [18].

In the study conducted by Khadkaa et al. (2019), the prevalence of poor sleep quality among 380 Nepali mothers within 2 to 12 months after their 
delivery was reported to be $28 \%$ [8]. In the study carried out by Hung et al, the mean score of the postpartum sleep quality scale of the participants was 22.82, indicating a mild level of sleep disorder. This study also showed a positive correlation between sleep quality and a satisfactory delivery experience and a negative one between that and the time spent doing household chores and activities on the daily basis [9] which was not consistent with the results of the present study, and the writer assumes this discrepancy may have been due to cultural, social and generally the sampling environment.

In another study conducted by Aksu et al. in Turkey, the mean score obtained by mothers in PSQS was 33.57, indicating mild sleep disorders in mothers at 2 to 8 weeks postpartum [1], which can be attributed to the tools which are different in 2 studies.

Factors such as age, parity, type of delivery, exclusive breastfeeding, body mass index, and cigarette smoking were not significantly different between women with good and bad sleep quality. Also, the study carried out in Canada in 2018 on mothers suffering from varying degrees of insomnia within 6 months postpartum showed that there was no statistically significant correlation between sleep disorders and infant sex, overnight breastfeeding, and infant sleep [11].

Likewise, in the study done by Behboudi Moqadam, there was no significant correlation between the frequency of pregnancy and sleep disorders [10], which was in consistent with the present study.

The study of Qassab Shirazi et al. (2013), done on women at the 2 and 6 weeks postpartum, showed that the group of women with normal vaginal delivery experienced a better sleep quality than those with cesarean section [5], which was not consistent with the results of this study, which might be due to the difference between the times at their postpartum, in that, the current study examined sleep quality of women being maximally 6 months postpartum, while Qassab Shirazi et al. did that of women maximally 6 weeks postpartum. In the Madrid-Valero study (2017), there was a direct correlation between body mass index and sleep quality [19], which was not consistent with the present study. The writer assumes this discrepancy can be attributed to the difference in the size of the populations of the two studies. In this study, anxiety level (pvalue $=0.002$ ) was found to be one of the most important variables affecting sleep quality in the postpartum period. Anxiety is a mental health disorder that can occur at any time. However, if it occurs at one year postpartum, it is called postpartum anxiety [20]. The results of the current study showed that $71.97 \%$ of people who experienced some degree of anxiety had poor sleep quality, as well. In the study of Clout et al., stress, anxiety and depression in women in the third trimester of pregnancy up to the 4-6 months postpartum were correlated with factors such as mother's sleep, health problems and baby's sleep and cesarean delivery(C-section) [21].

Likewise, Christian et al. (2018) in their study reported that insomnia and poor sleep quality were strongly correlated with symptoms of depression and anxiety in the postpartum period [22], which was consistent with the results of the present study.

Another variable examined in this study affecting the sleep quality of these women in the postpartum period was physical activity ( $\mathrm{p}$-value $=0.006$ ). $74.52 \%$ of mothers with low sleep quality had less than one hour of physical activity per week. Physical activity in the postpartum period has many health benefits such as improving sleep quality, reducing pregnancy weight, increasing and improving mood and reducing the risk of depression. Learning about factors such as sleep quality may enhance engagement in postnatal physical activity and provide recommendations for sedentary people to address the problem of inactivity in postpartum women.

In the study conducted by Sloan et al. (2020) in Singapore, there was a significant correlation between sleep quality and physical activity [23], which was consistent with this study. The study carried out by $\mathrm{Wu}$ et al., examining the relationship between sleep quality and duration of sleep in primiparous women at the 6 months postpartum and physical activity, showed that there was no significant correlation between sleep quality and 24-hour physical activity [18] The writer believes that this discrepancy may be due to the mere presence of primiparous mothers in that study.

There were several limitations in this study. The first one was the assessment of sleep quality 
which was relied solely on mothers' self-report. The second one was lack of information regarding mothers' sleep quality before their pregnancy. The third limitation was concerning examining the level of physical activity of mothers which was assessed merely by asking questions while it could be better to be done through a standard tool. And the last limitation was the cross-sectional nature of the study. Therefore, more and extended studies comparing sleep quality before and after pregnancy is needs to be made to generalize the results of the study to all postpartum women.

\section{Conclusion}

According to the findings of the present study and the fact that many physical and psychological problems in mothers and even their infants and other members of their family can be rooted in poor sleep quality, there is a need for measures other than the routine care done by healthcare forces for mothers to be taken which includes screening, training programs, and providing mothers with pamphlets and guidelines to diagnose sleep disorders and their related factors and help them with treatment and prevention.

\section{Acknowledgements}

The authors wish to thank the authorities of education and research departments and the ethics committee of the Zanjan University of Medical Sciences, and We would like to thank the participants in this research.

\section{Conflict of interest}

There is no conflict of interest to be declared.

\section{Authors' contributions}

All authors contributed to this project and article equally. All authors read and approved the final manuscript.

\section{References}

1. Aksu A, Vefikulucay Yilmaz D. The relationship of postpartum sleep quality and breastfeeding self-efficacy of Turkish mothers. Scand J Caring Sci. 2019; 33(4): 833- 39.

2. King LS, Rangel E, Simpson N, Tikotzky L, Manber R. Mothers' postpartum sleep disturbance is associated with the ability sensitivity. Sleep Med. 2020; 65: 74- 83.
3. Doering JJ, Dogan S. A Postpartum Sleep and Fatigue Intervention Feasibility Pilot Study. Behav. Sleep Med. 2018; 16(2):185- 201.

4. Lewis BA, Gjerdingen D, Schuver K, Avery M, Marcus BH. The effect of sleep pattern changes on postpartum depressive symptoms. BMC Womens Health. 2018; 18(12): 1- 7.

5. Ghasab Shirazi M, Raeesi Dehkor Z, Alidoosti $\mathrm{M}$, et al, Comparison of Maternal Sleep Quality in Postpartum Period between Vaginal Delivery and Cesarean Section. J Res Dev Nurs Midw. 2013; 10(1): 69- 76. [In Persian]

6. Yang Y, Li W, Ma TJ, et al. Prevalence of Poor Sleep Quality in Perinatal and Postnatal Women:

A Comprehensive Meta-Analysis of Observational Studies. Front Psychiatry. 2020; 11(161): 1- 10.

7. Nath S, Pearson RM, Moran P, et al. The association between prenatal maternal anxiety disorders and postpartum perceived and observed mother-infant relationship quality. J Anxiety Disord. 2019; 68: 102- 48

8. Khadka R, Hong SA, Chang YS. Prevalence and determinants of poor sleep quality and depression among postpartum women: a community-based study in Ramechhap district, Nepal. Int Health. 2020; 12(2): 125- 31.

9. Hung HM, Chen CH. Sleep Quality in Postpartum Women: Exploring Correlation With Childbirth Experience and Household Work. J Nurs Res. 2014; 22(1): 20- 27.

10. Behboodi Moghadam Z, Keshavarz Afshar M, Pourrahimi A, Taghizadeh Z, Mokhtari Zanjani P, Montazeri A. Evaluation of Postpartum Sleep Quality and the Influential Factors in the Women in Zanjan City, Iran (2014). Iran J Obstet Gynecol Infertil. 2018; 21(1): 6- 14. [In Persian]

11. Creti L, Libman E, Rizzo D, et al. Sleep in the Postpartum: Characteristics of First-Time, Healthy Mothers. Sleep Disord. 2017; 2017:1- 10. 12. Moghaddam JF, Nakhaee N, Sheibani V, Garrusi, B, Amirkafi A. Reliability and validity of the Persian version of the Pittsburgh Sleep Quality Index (PSQI-P). Sleep Breath. 2012; 16(1): 79- 82.

13. Pedram Razi SH, Varaei SH, Kazemnejad A, Bagheri F. The Effect of Foot Reflexology Massage on Sleep Quality in Women With Type 2 
Diabetes; a Clinical Study. Sci J Hamadan Nurs Midwifery Fac. 2018; 26(5): 283- 89.

14. Kaviani H, Mousavi AS. Psychometric properties of the Persian version of Beck Anxiety Inventory (BAI). Tehran Univ Med J. 2008; 66(2) :136- 40. [In Persian]

15. Taghavi F, Mirzaie P, Azandariani M, Rezaei Shojaei S.The Effectiveness of Short-Term Dynamic Psychotherapy on Anxiety, Depression and Happiness in Women with Generalized Anxiety. Med J Mashhad Univ Med Sci. 2020; 62: 456- 66.

16. Ko $\mathrm{SH}$, Chen $\mathrm{CH}$, Wang $\mathrm{HH}$, Yu-Ting Su. Postpartum women's sleep quality and its predictors in Taiwan. J Nurs Scholarsh. 2014; 46(2): 74- 81.

17. Wen SY, Ko YL, Chien LY. Sleep quality at three months postpartum considering maternal age: a comparative study. Women Birth. 2018; 31(6): 367- 73.

18. Wu J, Einerson B, Shaw JM, et al. Association between sleep quality and physical activity in postpartum women. Sleep Health. 2019; 5(6): 598- 605 .
19. Madrid-Valero JJ, Martínez-Selva JM, Ordoñana JR. Sleep quality and body mass index: a co-twin study. J Sleep Res. 2017; 26(4):461-67. 20. Wassif OM, Abdo AS, Elawady MA, Abd Elmaksoud AE, Eldesouky RS. Assessment of Postpartum Depression and Anxiety among Females Attending Primary Health Care Facilities in Qaliubeya Governorate, Egypt. J Environ Public Health. 2019; 2019: 1- 9.

21. Clout D, Brown R. Sociodemographic, pregnancy, obstetric, and postnatal predictors of postpartum stress, anxiety and depression in new mothers. J Affect Disord. 2015; 188: 60- 7.

22. Christian LM, Kowalsky JM, Mitchell AM, Porter K. Associations of postpartum sleep, stress, and depressive symptoms with LPSstimulated cytokine production among African American and White women. J Neuroimmunol. 2018; 316: 98106.

23. Sloan RA, Kim Y, Sawada SS, Asakawa A, Blair SN, Finkelstein EA. Is Less Sedentary Behavior, More Physical Activity, or Higher Fitness Associated with Sleep Quality? A CrossSectional Study in Singapore. Int J Environ Res Public Health. 2020; 17(4): 1- 11. 